\title{
Angle- and Energy-Resolved, Spin-Polarized Electron Emission Spectroscopy to Study Surface Magnetic and Electronic Properties
}

\author{
C. Rau, K. Waters, and N. Chen \\ Department of Physics and Rice Quantum Institute, Rice University, Houston, Texas 77251-1892
}

(Received 22 November 1989)

\begin{abstract}
We report on a new technique, spin-polarized electron emission spectroscopy. Using grazing-angle, surface scattering of $\mathrm{H}^{+}$and $\mathrm{He}^{+}$ions at $\mathrm{Ni}(110)$, we find that the angle-resolved energy distribution of emitted spin-polarized electrons is significantly different from that of electron-induced secondary (cascade) electrons and exhibits a series of characteristic "peaks" (including element-specific Auger electrons). We obtain characteristic, spin-dependent information on the surface electronic structure of $\mathrm{Ni}(110)$ and on atom-surface charge-transfer processes.
\end{abstract}

PACS numbers: $79.20 . \mathrm{Rf}, 61.14 . \mathrm{Rq}, 75.20 . \mathrm{En}$

Recently, broad and intense scientific interest has focused on the two-dimensional (2D) physical properties of surfaces of thin films and bulk materials. ${ }^{1,2}$ Grazing-ion surface reflection is a powerful means to probe these properties, in particular, the electronic, magnetic, and even chemical properties of surfaces. At grazing angles of incidence, ions do not penetrate a flat surface, they are specularly reflected and, therefore, probe the topmost surface layer. This is profitably exploited in electroncapture spectroscopy (ECS), 3,4 where capture of spinpolarized electrons by grazing-angle surface reflected deuterons is used to obtain information on surface magnetic behavior such as long- and short-ranged magnetic order, critical behavior, and 2D phase transitions. ${ }^{5}$

In recent years, a series of further spin-sensitive techniques has been devised and most successfully applied to study surface magnetism, ${ }^{2,6}$ but none of them fulfills all the requirements of simultaneously being extremely surface, spin, and element specific.

In this Letter, we report on a new and powerful method, energy-and angle-resolved, spin-polarized electron emission spectroscopy (SPEES), where grazingangle ion-surface reflection is used to induce the emission of spin-polarized electrons from the topmost layer of magnetic surfaces. Specifically the capability to detect element-specific, spin-polarized Auger electrons makes SPEES a very unique spectroscopy. Two other techniques are remotely related to SPEES: Electroninduced, spin-polarized Auger-electron spectroscopy (SPAES) successfully developed by Landolt, Allenspach, and $\mathrm{Mauri}^{7}$ and ion neutralization spectroscopy ${ }^{8}$ (INS) pioneered by Hagstrum and used to probe local electron density of states outside the surface.

An important aspect of SPEES is that it not only allows us to study the electronic, magnetic, and chemical structure of surfaces, but also enables us to unravel the physics of electronic charge-exchange processes occurring during particle surface interaction, which, at present, receives a great deal of attention. ${ }^{9-11}$

We employ the "spin" of emitted electrons as an additional "label" to identify various processes occurring in ion-surface interaction at grazing incidence. Selecting, for the incoming ions, grazing angles of incidence significantly helps to avoid cascade effects caused by energetic secondary electrons or ions and reveals important details about these processes.

In order to obtain a deeper insight into the specific physical processes underlying SPEES, we performed SPEES experiments at (110) surfaces of $\mathrm{Ni}$ pictureframe single crystals where the spin-polarized, groundstate surface electronic band structure is well known. ${ }^{1}$

Using SPEES at surfaces of well-defined, magnetized $\mathrm{Ni}(110)$ picture-frame single crystals, we find that the energy distribution of the grazing-angle, ion-induced $\left(\mathrm{H}^{+}\right.$or $\mathrm{He}^{+}$ions) emitted electrons is significantly different (absence of secondary-electron cascades) from that of electron-induced ${ }^{7}$ or ion-induced ${ }^{12}$ secondaryelectron spectra and shows pronounced energy-dependent features.

In angle- and energy-resolved SPEES, we use grazing-angle $\left(1^{\circ}\right)$ surface reflection of $15-30-\mathrm{keV} \mathrm{H}^{+}$- or $\mathrm{He}^{+}$-ion beams to study the emission of spin-polarized electrons as a measure of surface ferromagnetic order.

Figure 1 gives schematically the experimental setup used for SPEES. Using an einzellens system (2 in Fig. 1), we detect electrons emitted along the surface normal

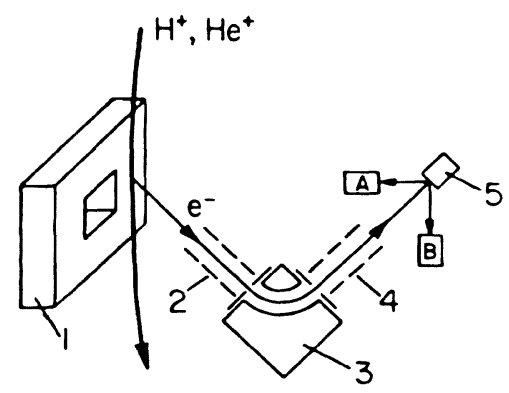

FIG. 1. Experimental setup used in SPEES experiments: 1, magnetized $\mathrm{Ni}(110)$ picture-frame single crystal; 2 and 4 , einzellenses; 3 , electrostatic energy analyzer; 5, electron-spin detector with Au-Al target and two channeltrons $\boldsymbol{A}$ and $\boldsymbol{B}$. 
(emission cone angle $11^{\circ}$ ) of a remanently magnetized target [Ni(110) picture-frame single crystal; 1 in Fig. 1]. For energy analysis, an electrostatic energy analyzer is used ( 3 in Fig. 1). For an energy resolution of $300 \mathrm{meV}$, using a channeltron, the count rates amount to about $10 \%$ s.

For spin analysis, using a second einzellens system (4 in Fig. 1), the energy-analyzed electrons are accelerated to $150 \mathrm{eV}$ and enter a precisely calibrated low-energy electron-spin detector which allows for a fast and efficient determination of the electron-spin polarization (ESP). In this ESP detector, the electron beam is precisely focused onto a Au target ( $\approx 2 \mathrm{~mm}$ in diameter) certifying that the measured count ratios $N_{A} / N_{B}$ of electrons backscattered in two channeltrons $A$ and $B$ (see Fig. 1), positioned at $135^{\circ}$ to the incoming beam direction, depend only on counting statistics. $N_{A} / N_{B}$ provides a direct measure of the long-ranged ESP. We refer to Ref. 13 for further details on this ESP detector. For zero-ESP calibration, the $\mathrm{Au}$ target is replaced by an $\mathrm{Al}$ target.

Zero-ESP calibration can also be performed by replacing the ferromagnetic Ni target by a nonmagnetic sample such as $\mathrm{Cu}$ where, as expected, over the whole investigated energy range, zero ESP is detected. Zero-ESP values are also found when the ESP measurements are taken far above the Curie temperature of $\mathrm{Ni}$ where $\mathrm{Ni}$ exhibits a loss of long-ranged surface ferromagnetic order.

A magnetizing field (along the [111] direction in the (110) surface plane of a $\mathrm{Ni}(110)$ picture-frame single crystal) is applied in the surface plane of the sample to magnetically saturate the specimen. With $P$, the ESP along the magnetizing field, we obtain $P=\left(n^{+}-n^{0}\right) /$ $\left(n^{+}+n^{-}\right)$, where $n^{+}$and $n^{-}$are fractional numbers of electrons emitted with moment parallel (majority-spin electrons) and antiparallel (minority-spin electrons), respectively, to the applied field.

The samples are prepared and characterized in a target preparation chamber at $1 \times 10^{-10} \mathrm{mbar}$ as described in Ref. 4 and then transferred to the SPEES measurement chamber operating at present at a working pressure of $9 \times 10^{-10}$ mbar where $90 \%$ of the residual gas consists of hydrogen. In order to be able to show that SPEES is sensitive to residual surface contaminants, the cleaning of the Ni(110) surfaces, using argon-ion sputtering, was terminated when a residual $\mathrm{C}$ and $\mathrm{O}$ surface coverage of a few percent was reached.

Figures 2(a) and 2(b) show the spin $P(E)$ and the intensity $I(E)$ distribution of spin-polarized electrons emitted during grazing-angle $\left(1^{\circ}\right)$ surface reflection of 25 $\mathrm{keV} \mathrm{H}{ }^{+}$ions at magnetized $\mathrm{Ni}(110)$ surfaces at $300 \mathrm{~K}$ as functions of the electron energy $E$.

The distance $d_{\min }$ of closest approach of the ions towards the reflecting surface is well characterized by the energy component $E_{\perp}$ of the ions normal to the surface $E_{\perp}=E_{0} \sin ^{2} \alpha \approx E_{0} \alpha^{2}$. With $E_{0}=25 \mathrm{keV}, E_{\perp}$ amounts
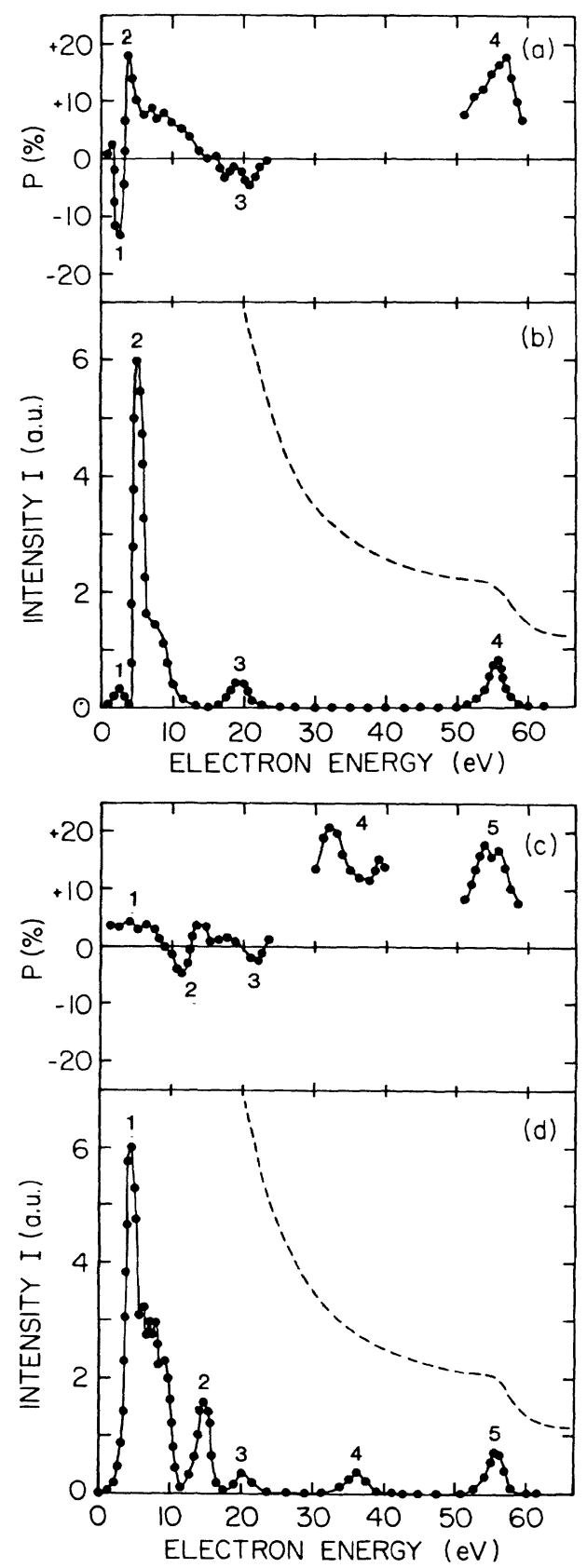

FIG. 2. Intensity distribution $I(E)$ (energy resolution $\Delta E$ $=0.3 \mathrm{eV}$ ) and electron-spin polarization $P(E)$ (experimental error $\Delta P=+2 \%$ ) of spin-polarized electrons emitted during grazing-angle surface reflection of (a),(b) $25-\mathrm{keV}$ protons and (c), (d) $25-\mathrm{keV} \mathrm{He}^{+}$ions at magnetized $\mathrm{Ni}(110)$ as functions of the electron energy $E$.

to $7.6 \mathrm{eV}$ for a reflection angle $\alpha=1^{\circ}$. Using planar surface potentials for $\mathrm{Ni}(110)$ (see Fig. 9 in Ref. 4), $d_{\text {min }}$ amounts to $0.1 \mathrm{~nm}$ for $\alpha=1^{\circ}$ which reveals that under these experimental conditions the ions cannot penetrate the Ni(110) surface.

Replacing, for surface reflection at $\mathrm{Ni}(110)$, the 25$\mathrm{keV} \mathrm{H}^{+}$ions by $25-\mathrm{keV} \mathrm{He}^{+}$ions, results in the $I(E)$ and $P(E)$ data given in Figs. 2(c) and 2(d). As for $\mathrm{H}^{+}$, 
we use $\alpha=1^{\circ}$ to prevent penetration of the helium ions into deeper surface layers. We observe four lowintensity peaks [located at $14,20,36$, and $56 \mathrm{eV}$; see labels 2, 3, 4, and 5 in Fig. 2(d)] and one high-intensity peak [see label 1 in Fig. 2(d)] located with a maximum at around $4.5 \mathrm{eV}$.

From Figs. 2(b) and 2(d), it is obvious that $I(E)$ is completely different from that of electron-induced secondary-electron spectra [see dashed lines ${ }^{7}$ in Figs. $2(\mathrm{~b})$ and $2(\mathrm{~d})]$. We note that the use of large angles of incidence for the incoming ions, where the particles can deeply penetrate the surface, prevents the occurrence of such distinct peaks.

From Fig. 2(b), we observe three low-intensity peaks [located at 2.5-3, 20, and $56 \mathrm{eV}$; see labels 1,3 , and 4 in Fig. 2(b)] and one high-intensity peak [see label 2 in Fig. 2(b)] located with a maximum at around $4.5 \mathrm{eV}$. By varying the primary beam energy of the $\mathrm{H}^{+}$ions between 15 and $30 \mathrm{keV}$, we observe no measurable shift in the energetic location of these peaks.

This gives first evidence to exclude, for the interpretation of our data, models based on kinetic electron ${ }^{14}$ or convoy electron emission ${ }^{15}$ and points towards electron emission in terms of various $X V V$ Auger transitions (see Fig. 3) ${ }^{8}$ We denote by $X$ an electronic level either of the incoming ion or of atoms located at the surface with valence-band levels denoted by $V$.

The corresponding spin distributions $P(E)$ of the emitted electrons [see Figs. 2(a) and 2(c)] show several pronounced, characteristic peaks [see labels 1-4 in Fig. 2(a) and labels 2-5 in Fig. 2(c)].

In the following, we use the sign and magnitude of the ESP as additional "labels" to identify the underlaying charge-exchange processes. We further assume that $X V V$ Auger peaks, where $X$ is a level of the incoming ion, should undergo a shift in energy when the $\mathrm{H}^{+}$ions are replaced by $\mathrm{He}^{+}$ions. $X V V$ Auger transitions, where $X$ is an empty electronic level of a surface atom, should not undergo such a shift.

From theoretical $\mathrm{Ni}(110)$ ground-state, one-electron band-structure calculations, ${ }^{1}$ from ECS experiments, ${ }^{3,4}$ and from other spin-sensitive experiments at $\mathrm{Ni}(110)$ surfaces, ${ }^{2,6,16,17}$ it is known that $V$-band electrons, originating from energy levels near $(\$ 0.5 \mathrm{eV})$ the Fermi energy, possess a predominant minority-spin orientation (negative ESP) of nearly $-100 \%$, whereas $V$ electrons from energy levels below this energy range overwhelmingly possess a majority-spin orientation (positive ESP). We note that from high-resolution, spin-polarized photoemission experiments ${ }^{16,17}$ it is found that for $\mathrm{Ni}(110)$ the negative ESP at photothreshold becomes already positive at a crossover energy of $0.32 \mathrm{eV}$ which is around half of the value predicted by band-structure theory. ${ }^{1}$

This information can be profitably used to identify and unravel the nature of the various physical processes involved in the electron emission in SPEES. For instance, $V$ electrons originating in $k$ space from energy levels lo-
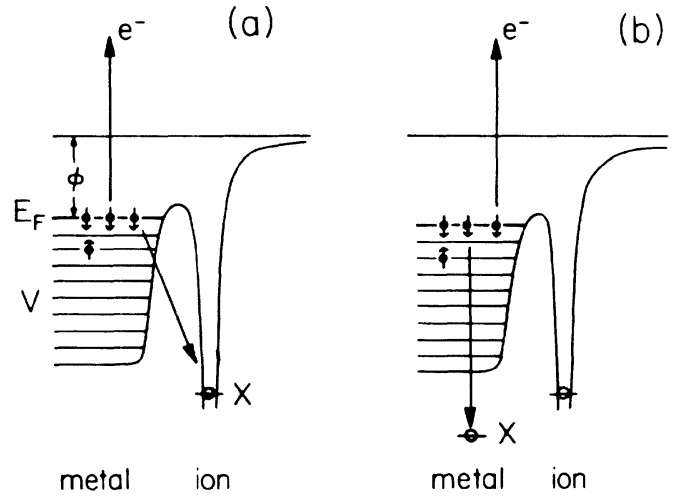

FIG. 3. Auger-type, two-electron charge-transfer processes for $\mathrm{a} \mathrm{H}^{+}$or $\mathrm{He}^{+}$ion near a solid surface. Process (a): $X V V$ Auger transition with $X$ being the ground-state $(1 s)$ energy level of the ion. Process (b): $X V V$ Auger transition with $X$ being an ion-induced hole of a surface atom. $V$ denotes valenceband levels, $\phi$ the work function, and $E_{F}$ the Fermi energy of the metal.

cated $0.5 \mathrm{eV}$ or less near the Fermi level, being emitted without any spin-flip process involved, would contribute to the negative part of the $P(E)$ curve. On the other hand, $V$ electrons originating from levels located at least 0.3-0.5 eV below the Fermi level would contribute to the positive part of the $P(E)$ curve.

Figure 3 illustrates two possible two-electron chargeexchange processes in terms of $X V V$ Auger neutralization. Process (a): neutralization of an incoming hydrogen or helium ion at a metal surface and emission of an Auger electron. Process (b): $X V V$ Auger-electron emission where $X$ is, produced by the incoming ion, a hole either in the valence $(V)$ bands or lower levels of the surface atoms.

In a first qualitative attempt, we discuss several $X V V$ Auger transitions which could occur near $\mathrm{Ni}(110)$ surfaces and contribute to the emission of spin-polarized electrons in SPEES.

Process (a): $X V V$ Auger transition in which $X$ is a level of the incoming ion. For the work function $\phi$ of $\mathrm{Ni}(110)$, we use a value of $5.04 \mathrm{eV}$ (Ref. 18) and for the $1 s$ ground state of hydrogen a value of $E_{X}=-13.58 \mathrm{eV}$ which gives a maximum kinetic energy of the ejected Auger electrons of $13.58-(2 \times 5.05) \mathrm{eV}=3.5 \mathrm{eV}$. [Note that dynamic screening of the $1 s$ hole of the incoming proton by the electrons at the $\mathrm{Ni}(110)$ surface would decrease the binding energy of the hydrogen $1 s$ bound state and consequently reduce the maximum kinetic energy of the ejected Auger electron to slightly lower values.] Qualitatively taking into account the band structure of Ni(110), $P(E)$ should first exhibit a negative value at around $3 \mathrm{eV}$ followed by an immediate change to positive $P$ values with decreasing electron energy. This is indeed observed in the experiment. Using an energy resolution of $0.3 \mathrm{eV}$, we observe [see peak 1 in Fig. 2(a)] a change from $-13 \%$ to $+2 \%$ within $0.7 \mathrm{eV}$. For com- 
parison, in spin-polarized photoemission experiments, $P$ amounts to $-52 \%$ (Ref. 16) or $-100 \%$ (Ref. 17) at photothreshold, reaches zero within $0.3 \mathrm{eV}$, and increases to $+20 \%$ within $0.5 \mathrm{eV}$.

Replacing the $\mathrm{H}^{+}$ions by $\mathrm{He}^{+}$ions should cause an energetic shift of this peak [peak 1 in Fig. 2(b)] by 11 $\mathrm{eV}$ which is the difference between the $\mathrm{He}^{+}(1 s)\left(E_{X}\right.$ $=-24.6 \mathrm{eV}$. $)^{8}$ and $\mathrm{H}^{+}(1 s)$ ground-state energies. This is indeed observed in the experiment [peak 2 in Fig. 2(d)] and further corroborated by the occurrence of a change of $P$ from negative $(-5 \%)$ to positive values $(+5 \%)$ [see peak 2 in Fig. $2(\mathrm{c})$ ] as discussed before for the $\mathrm{H}^{+}$data.

We note that the electron peak located at around 36 eV [see peak 4 in Fig. 2(c)] was already observed by Hagstrum and Becker ${ }^{8}$ and quite recently by Niehaus and co-workers ${ }^{19}$ and was interpreted by the latter group as a two-electron-capture process from $\mathrm{He}^{++}-\mathrm{Cu}(110)$ collisions leading to $\mathrm{He}^{* *}\left(2 p^{2}\right)$ decaying to $\mathrm{He}^{+}(1 s)$ followed by the emission of an electron of $36-\mathrm{eV}$ kinetic energy. We remark that in the present experiments a residual part of the ionic $\mathrm{He}$ beam consists of $\mathrm{He}^{++}$ions.

Process (b): $X V V$ Auger transitions in which, induced by the incoming ion, $X$ is a spin-polarized hole in the valence or lower electronic levels of the surface $\mathrm{Ni}$ or impurity atoms. Comparing Figs. 2(b) and 2(d), we observe that the three peaks located at around 4.5, 20, and $56 \mathrm{eV}$ [see peaks 2, 3, and 4 in Fig. 2(b) and peaks 1, 3, and 5 in Fig. 2(d)] are independent of the ion species used in the experiments and are not shifted to higher energies by the use of $\mathrm{He}^{+}$ions instead of $\mathrm{H}^{+}$ions. Therefore, we attribute these peaks to Auger transitions where the ions are solely used to produce ion-induced holes in the electron shells of the surface atoms.

Comparing our data with presently existing Augerelectron data for $\mathrm{Ni}$ and with data obtained by Landolt, Allenspach, and Mauri ${ }^{7}$ using spin-polarized Augerelectron spectroscopy at $\mathrm{Ni}(100)$ surfaces, we identify the peak located at $56 \mathrm{eV}$ [see peak 4 in Fig. 2(b) and peak 5 in Fig. 2(d)] with a $M_{23} V V\left(M_{23} M_{45} M_{45}\right) \mathrm{Ni}$ Auger decay. We note that, after background subtraction, these authors find similar $P$ values for this peak.

Regarding the peak [see label 3 in Figs. 2(b) and 2(d)] located at around $20 \mathrm{eV}$, we remark that the intensity of this peak depends strongly on surface cleanness, and, therefore, can be attributed to Auger decays where $X$ is, induced by the incoming ions, a hole level in the surface contaminants.

Because of the similarities of the electron spectra [see peak 2 in Fig. 2(b) and peak 1 in Fig. 2(d)] at around $4.5 \mathrm{eV}$, induced by $\mathrm{H}^{+}$and $\mathrm{He}^{+}$, it is tempting to correlate this feature with the substrate. A possible mechanism could be an ion-induced, kinematically enhanced $X V V$ Ni Auger decay with $X$ the level in the lower part of the valence band. Because of the differences in the polarization $(P=18 \%$ vs $P=4 \%$ at $4.5 \mathrm{eV})$ such a clas- sification is, however, not as clear as for the $M_{23} M_{45} M_{45}$ $\mathrm{Ni}$ peak. The similarity of this peak with the secondary-electron cascade generated by sputtering ${ }^{12}$ suggests this mechanism as a possible origin of the peak.

In conclusion, we note that, provided the future availability of a quantitative theory of SPEES and more refined experimental data, SPEES will not only advance towards an experimental technique of great potential for studies on the spin-polarized, local electronic structure of the topmost surface layer, it also can provide very stimulating and detailed data on the particle-solid-interaction mechanism.

We thank H. Hopster and P. Nordlander for stimulating discussion. This work is supported by the National Science Foundation, by the Welch Foundation, and by the Texas Higher Education Coordinating Board.

\footnotetext{
${ }^{1}$ H. Krakauer, A. J. Freeman, and E. Wimmer, Phys. Rev. B 28, 610 (1983); C. L. Fu, A. J. Freeman, and T. Oguchi, Phys. Rev. Lett. 54, 2700 (1985).

${ }^{2}$ Magnetic Properties of Low-Dimensional Systems, edited by L. M. Falicov and J. L. Moràn-López, Springer Proceedings in Physics Vol. 14 (Springer-Verlag, Berlin, 1986).

${ }^{3}$ C. Rau and R. Sizmann, Phys. Lett. 43A, 317 (1973).

${ }^{4}$ C. Rau, J. Magn. Magn. Mater. 30, 141 (1982).

${ }^{5}$ C. Rau, Appl. Phys. A 49, 579 (1989).

${ }^{6}$ F. B. Dunning, C. Rau, and G. K. Walters, Comments Solid State Phys. 12, 17 (1985); J. Kirschner, K. Koike, and H. P. Oepen, Phys. Rev. Lett. 59, 2099 (1987); H. Winter, H. Hagedorn, R. Zimny, H. Nienhaus, and J. Kirschner, Phys. Rev. Lett. 62, 296 (1989).

${ }^{7}$ M. Landolt, R. Allenspach, and D. Mauri, J. Appl. Phys. 57, 3626 (1985).

${ }^{8}$ H. D. Hagstrum and G. E. Becker, Phys. Rev. B 8, 107 (1973).

${ }^{9}$ P. Nordlander and J. C. Tully, Phys. Rev. Lett. 61, 990 (1988).

${ }^{10}$ D. M. Newns, Comments Condens. Matter Phys. 14, 225 (1989).

${ }^{11}$ N. D. Lang, Phys. Rev. B 27, 2019 (1983).

${ }^{12}$ G. Wehner, Z. Phys. 193, 439 (1966).

${ }^{13}$ J. Unguris, D. T. Pierce, and R. J. Celotta, Rev. Sci. Instrum. 59, 1314 (1986).

$14 \mathrm{~J}$. Schou, Nucl. Instrum. Methods 170, 317 (1980).

${ }^{15}$ M. Breinig, S. B. Elston, S. Huldt, L. Liljeby, C. R. Vane, S. D. Berry, G. A. Glass, M. Schauer, I. A. Sellin, G. D. Alton, S. Datz, S. Overbury, R. Laubert, and M. Suter, Phys. Rev. A 25, 3015 (1981).

${ }^{16}$ W. Gudat, E. Kisker, E. Kuhlmann, and M. Campagna, Phys. Rev. B 22, 3282 (1980).

${ }^{17}$ E. Kisker, W. Gudat, E. Kuhlmann, R. Clauberg, and M. Campagna, Phys. Rev. Lett. 45, 2053 (1980).

${ }^{18}$ B. G. Baker, B. B. Johnson, and G. L. C. Maire, Surf. Sci. 24, 572 (1971).

${ }^{19}$ P. A. Zeiglinans Van Emmichoven, P. A. A. F. Wouters, and A. Niehaus, Surf. Sci. 195, 115 (1988).
} 